\title{
Maximum Norm Estimates of the Solution of the Navier-Stokes Equations in the Halfspace with Bounded Initial Data
}

\author{
Santosh Pathak \\ Department of Mathematics, University of Utah Asia Campus, 119-3 Songdo Moonhwa-Ro Yeonsu-Gu, Incheon, Republic of Korea \\ 21985 \\ Correspondence should be addressed to Santosh Pathak; s.pathak@utah.edu
}

Received 4 January 2021; Accepted 6 February 2021; Published 17 February 2021

Academic Editor: Janne Heittokangas

Copyright (C) 2021 Santosh Pathak. This is an open access article distributed under the Creative Commons Attribution License, which permits unrestricted use, distribution, and reproduction in any medium, provided the original work is properly cited.

\begin{abstract}
In this paper, I consider the Cauchy problem for the incompressible Navier-Stokes equations in $\mathbb{R}_{+}^{n}$ for $n \geq 3$ with bounded initial data and derive a priori estimates of the maximum norm of all derivatives of the solution in terms of the maximum norm of the initial data. This paper is a continuation of my work in my previous papers, where the initial data are considered in $\mathbb{T}^{n}$ and $\mathbb{R}^{n}$ respectively. In this paper, because of the nonempty boundary in our domain of interest, the details in obtaining the desired result are significantly different and more challenging than the work of my previous papers. This challenges arise due to the possible noncommutativity nature of the Leray projector with the derivatives in the direction of normal to the boundary of the domain of interest. Therefore, we only consider one derivative of the velocity field in that direction.
\end{abstract}

\section{Introduction}

We consider the Cauchy problem of the incompressible Navier-Stokes equations in $\mathbb{R}_{+}^{n}, n \geq 3$ :

$$
\begin{array}{lll}
u_{t}+u \cdot \nabla u+\nabla p & =\Delta u \text { for } x \in \mathbb{R}_{+}^{n}, t>0 \\
\nabla \cdot u & =0 \text { for } x \in \mathbb{R}_{+}^{n}, t>0 \\
\left.u\right|_{t=0} & =f \text { for } x \in \mathbb{R}_{+}^{n} \\
\left.u\right|_{x_{n}=0} & =0 \text { for } t>0
\end{array}
$$

where $u=u(x, t)=\left(u_{1}(x, t), \cdots, u_{n}(x, t)\right)$ and $p=p(x, t)$ stand for the unknown velocity vector field of the fluid and its pressure, while $f=f(x)=\left(f_{1}(x), \cdots f_{n}(x)\right)$ is the given initial velocity vector field, with $\nabla \cdot f=0$ and $\left.f\right|_{x_{n}=0}=0$. In what follows, we will use the same notations for the space of vectorvalued and scalar functions for convenience in writing.

There is a large literature on the existence and uniqueness of solution of the Navier-Stokes equations in $\mathbb{R}^{n}$. For the given initial data, solutions of (1) have been constructed in various function spaces. For example, if $f \in L^{r}$ for some $r$ with $3 \leq r<\infty$, then it is well known that there is a unique classical solution in some maximum interval of time: $0 \leq t<T_{f}$, where $0<T_{f} \leq \infty$. But, for the uniqueness of the pressure, one requires $|p(x, t)| \longrightarrow 0$ as $|x| \longrightarrow \infty$. See [1] and [2] for $r=3$ and [3] for $3<r<\infty$. The solution is $C^{\infty}$ for $0<T_{f}<\infty$.

It is well known that for $f \in L^{\infty}\left(\mathbb{R}^{n}\right)$, there is a unique, smooth, and local-in-time solution $u$ for the Navier-Stokes equations with

$$
p=\sum_{i, j} R_{i} R_{j} u_{i} u_{j}
$$

where $R_{i}=(-\Delta)^{-1 / 2} \partial_{x_{i}}$ is the $i$ th Riesz operator. It is known that in $\mathbb{R}^{2}$, this solution can be extended globally in time. For $f \in L^{\infty}\left(\mathbb{R}^{n}\right)$, where $n \geq 3$, the existence of a regular solution follows from [4]. The solution is only unique if one puts some growth restrictions on the pressure as $|x| \longrightarrow \infty$. A simple example of nonuniqueness is demonstrated in [5], where the velocity $u$ is bounded, but $|p(x, t)| \leq C|x|$. In addition, an estimate $|p(x, t)| \leq C\left(1+|x|^{\sigma}\right)$ with $\sigma<1$ (see [6]) implies uniqueness. Also, the assumption $p \in L_{l o c}^{1}(0, T$; BMO) (see [7]) implies uniqueness. 
For $f \in L^{\infty}\left(\mathbb{R}_{+}^{n}\right)$, where $n \geq 3$, the existence of a local mild solution is proved by Bae and Jin in [8]. In the same paper, it is also proved that such mild solution is indeed a strong solution of the Navier-Stokes equations (1). Before the result of Bae and Jin, the local-in-time existence of mild (strong) solution of the halfspace problem was provided in [9] by Solonikov for continuous bounded initial data in $\mathbb{R}_{+}^{n}$.

In this paper, I am interested in obtaining estimates of the maximum norm of the derivatives of $u$ in terms of the maximum norm of the initial function $f$, assuming that the solution exists, and it is $C^{\infty}\left(\mathbb{R}_{+}^{n}\right)$ for $0<t<T_{f}$. The work of this paper is a continuation of the work of my papers [10] and [11] to the halfspace case for nondecaying initial data. Nonempty boundary in the domain in this paper makes this work different, in some aspects, and significantly more challenging in proving the key lemmas than the work in my previous works where the initial functions are in $\mathbb{R}^{n}$ or $\mathbb{T}^{n}$.

We begin by transforming the momentum equations of (1) into the abstract ordinary differential equations:

$$
u_{t}+A u=-\mathbb{P}(u \cdot \nabla u)
$$

where $A=-\mathbb{P} \Delta$ is the Stokes operator and $\mathbb{P}$ is the Leray projector, which is given by

$$
\mathbb{P} f(x) \equiv f(x)+\nabla_{x} \int_{\mathbb{R}_{+}^{n}} \nabla_{y} \mathscr{G}(x, y) \cdot f(y) d y,
$$

where $\left.f_{n}\right|_{x_{n}=0}=0$. Note that

$$
\mathscr{G}(x, y) \equiv N(x-y)+N\left(x-y^{*}\right)
$$

where $y^{*}=\left(y_{1}, \cdots y_{n-1},-y_{n}\right), N(x)=\left(1 /(2-n) \omega_{n}\right)|x|^{2-n}$, if $n$ $\geq 3$, and $\omega_{n}$ denotes the surface area of the unit sphere in $\mathbb{R}^{n}$ which is given by $\omega_{n}=2 \pi^{n / 2} / \Gamma(n / 2)$. form:

The solution of (3) is formally expressed in the integral

$$
u(t)=e^{-A t} f-\int_{0}^{t} e^{-A(t-s)} \mathbb{P}(u \cdot \nabla u)(s) d s
$$

Solonikov [9] has expressed the solution operator of the Stokes equations in $\mathbb{R}_{+}^{n}$ in the integral form

$$
e^{-A t} f \equiv \int_{\mathbb{R}_{+}^{n}} G(x, y, t) \cdot f(y) d y,
$$

where $G=\left(G_{i j}\right)_{i, j=1, \cdots n}$ is given by

$$
\begin{aligned}
G_{i j}(x, y, t)= & \delta_{i j}\left(\Gamma(x-y, t)-\Gamma\left(x-y^{*}, t\right)\right) \\
& +4\left(1-\delta_{j n}\right) \frac{\partial}{\partial x_{j}} \int_{0}^{x_{n}} \int_{\mathbb{R}^{n-1}} \frac{\partial}{\partial x_{i}} N(x-z) \\
& \cdot \Gamma\left(z-y^{*}, t\right) d z .
\end{aligned}
$$

The function $\Gamma(x, t)$ is the $n$-dimensional Gaussian kernel defined by $\Gamma(x, t) \equiv \Gamma_{t}(x) \equiv\left(1 /(4 \pi t)^{n / 2}\right) e^{-|x|^{2} / 4 t}$.
A solution formula of the Stokes equations (3) in $\mathbb{R}_{+}^{n}$ has also been provided by Ukai in [12]. Such solution formula has been used in the $L^{q}$ setting, particularly for $1<q<\infty$ (see $[13,14])$. For $L^{1}$ and $L^{\infty}$ estimates of the Stokes flow or its gradient, see $[15,16]$. The solution formula provided by Solonikov [9] has mainly been used for $L^{\infty}$ framework (see $[14,17])$.

To formulate the main result of this paper, we first introduce some notations as follows:

$$
|f|_{\infty}=\sup _{x}|f(x)| \text { with }|f(x)|^{2}=\sum_{i} f_{i}^{2}(x)
$$

and $D^{\alpha}=D_{1}^{\alpha_{1}} \cdots D_{n}^{\alpha_{n}}, \partial x_{i}=D_{i}=\left(\partial / \partial x_{i}\right)$ for a multi - index $\alpha$ $=\left(\alpha_{1}, \cdots, \alpha_{n}\right)$. In what follows, if $|\alpha|=j$, for any $j=0,1, \cdots$, then we will denote $D^{\alpha}=D_{1}^{\alpha} \cdots D_{n}^{\alpha_{n}}$ by $D^{j}$. We also set

$$
\left|\mathscr{D}^{j} u(t)\right|_{\infty}:=\left|\mathscr{D}^{j} u(\cdot, t)\right|_{\infty}=\max _{|\alpha|=j}\left|D^{\alpha} u(\cdot, t)\right|_{\infty} \cdot
$$

Clearly, $\left|\mathscr{D}^{j} u(t)\right|_{\infty}$ measures all space derivatives of order $j$ in maximum norm. For later purposes, let us also introduce a few other notations:

$$
\begin{gathered}
\Gamma(x, t)=\Gamma_{t}(x)=\bar{\Gamma}_{t}(\bar{x}) \Gamma_{t}^{n}\left(x_{n}\right) \\
=\left(\frac{1}{(4 \pi t)^{(n-1) / 2}} e^{-|\bar{x}|^{2} / 4 t}\right)\left(\frac{1}{(4 \pi t)^{1 / 2}} e^{-x_{n}^{2} / 4 t}\right), \\
D^{\beta}=\left(D_{1}^{\beta_{1}} \ldots, D_{n-1}^{\beta_{n-1}}\right), D^{\alpha}=D^{\beta} D_{n}, \quad \alpha=(\beta, 1), \quad \nabla \cdot=\operatorname{div} .
\end{gathered}
$$

Throughout this paper, $D^{j}$ will be understood as the derivative of order $j=|\alpha|=|\beta|+1$. In addition, $1_{\mathbb{R}_{+}^{n}}$ denotes the characteristic function which is 1 on $\mathbb{R}_{+}^{n}$ and 0 otherwise. $\tau_{a}$ is a translation operator defined by $\tau_{a} f(x):=f(a-x)$.

The goal of this paper is to prove the following theorem.

Theorem 1. Consider the Cauchy problem for the NavierStokes equations ((1)) where $f \in L^{\infty}\left(\mathbb{R}_{+}^{n}\right)$ and $\nabla \cdot f=0$ is understood in the sense of distribution. There is a constant $c_{0}>0$, and for any $\alpha=\left(\alpha_{1}, \cdots \alpha_{n-1}, 1\right)$ with $|\alpha|=j$ where $j=$ $0,1, \cdots$ there is a constant $K_{j}$ so that

$$
t^{j / 2}\left|\mathscr{D}^{j} u(t)\right|_{\infty} \leq K_{j}|f|_{\infty} \quad \text { for } \quad 0<t \leq \frac{c_{0}}{|f|_{\infty}^{2}}
$$

The constants $c_{0}$ and $K_{j}$ are independent of $t$ and $f$.

One of the important tools in the proof of Theorem 1 is the uniform estimates of the composite operator $D^{j} e^{-A t} \mathbb{P} \nabla$ div. But, obtaining such uniform estimates is complicated because of the possible noncommutativity nature of the Leray projector with the derivatives in the direction of normal to the boundary of the domain; hence, $D^{j}$ and $e^{-A t} \mathbb{P}$ may not be commutative. 
To overcome this difficulty, we will generalize the techniques of obtaining the uniform estimates on $\nabla e^{-A t} \mathbb{P} \nabla$ div of the paper [8] by Bae and Jin to obtain our desired uniform estimates on $D^{j} e^{-A t} \mathbb{P} \nabla$ div. In their paper, they require the uniform estimates to prove the existence of the local solution of the Navier-Stokes equations in halfspace for bounded initial data.

This paper is organized in the following ways. In "Some Auxiliary Results," we introduce some auxiliary results which will be labelled as propositions. In "Estimate of $\mathrm{D}^{\mathrm{j}} \mathrm{e}^{-\mathrm{At}} \mathrm{P} \nabla \cdot \mathrm{g}$," we derive an important estimate on the composite operator $D^{j} e^{-A t} \mathbb{P} \nabla$ div. In "Estimates for the Navier-Stokes Equations," we establish some estimates on the solution of the Navier-Stokes equations. In "Estimates for the NavierStokes Equations," a proof of Theorem 1 will be provided. Finally, Appendices A, B, and C contain proofs of the propositions which are introduced in "Some Auxiliary Results."

\section{Some Auxiliary Results}

Let us consider the Stokes problem in $\mathbb{R}_{+}^{n}, n \geq 3$ :

$$
\left.\begin{array}{lll}
u_{t}-\Delta u+\nabla p & =-\nabla \cdot g(u) \text { for } x \in \mathbb{R}_{+}^{n}, t>0, \\
\nabla \cdot u & =0 \text { for } x \in \mathbb{R}_{+}^{n}, t>0, \\
\left.u\right|_{t=0} & =f \text { for } x \in \mathbb{R}_{+}^{n}, \\
\left.u\right|_{x_{n}=0} & =0 \text { for } t>0,
\end{array}\right\}
$$

where $g=u \otimes u=\left(g_{i j}\right)_{1 \leq i, j \leq n}$. Here, we note that each $g_{i j}$ is quadratic in components of $u$.

Solonikov in [9] has obtained the solution of (13) which is given by

$$
\begin{aligned}
u(x, t)= & \int_{\mathbb{R}_{+}^{n}} G(x, y, t) \cdot f(y) d y \\
& -\int_{0}^{t} \int_{\mathbb{R}_{+}^{n}} G(x, y, t-s) \cdot(\mathbb{P} \nabla \cdot g)(y, s) d y d s .
\end{aligned}
$$

Next, we state the following proposition.

Proposition 2. If $k=1, \cdots, n-1$, then we have

$$
\frac{\partial}{\partial x_{k}} G_{i j}(x, y, t)=-\frac{\partial}{\partial y_{k}} G_{i j}(x, y, t)
$$

and

$$
\begin{aligned}
\frac{\partial}{\partial x_{n}} G_{i j}(x, y, t)= & \frac{\partial}{\partial y_{n}} G_{i j}(x, y, t)-2 \delta_{i j} \frac{\partial}{\partial y_{n}} \Gamma(x-y, t) \\
& +4\left(1-\delta_{j n}\right) \frac{\partial}{\partial x_{j}} \int_{\mathbb{R}^{n-1}} \frac{\partial}{\partial x_{i}} N\left(\bar{x}-\bar{z}, x_{n}\right) \\
& \cdot \Gamma\left(\bar{z}-\bar{y}, y_{n}, t\right) d \bar{z} .
\end{aligned}
$$

Proof. The proof is given in Appendix A.
Proposition 3. Let $x \in \mathbb{R}_{+}^{n}$ and $f$ be any Hölder continuous function with the exponent $0<\alpha<1$ :

$$
[f]_{\alpha}=\sup _{x, z} \frac{|f(x)-f(z)|}{|x-z|^{\alpha}}<\infty .
$$

Then, for $i, j \neq n$ or $i, j=n$, we have

$$
\begin{aligned}
& \partial_{x_{j}} \int_{0}^{x_{n}} \int_{\mathbb{R}^{n-1}} \partial_{x_{i}} N(x-z) f(z) d z=-\frac{\delta_{i j}}{2 n} f(x) \\
& \quad+\int_{0}^{x_{n}} \int_{\mathbb{R}^{n-1}} \partial_{x_{i} x_{j}}^{2} N(x-z) f(z) d z \\
& \quad+\delta_{j n} \int_{\mathbb{R}^{n-1}} \partial_{x_{i}} N(\bar{x}-\bar{z}) f\left(\bar{z}, x_{n}\right) d \bar{z} .
\end{aligned}
$$

For $i=n, j \neq n$ or $j=n, i \neq n$, we have

$$
\partial_{x_{j}} \int_{0}^{x_{n}} \int_{\mathbb{R}^{n-1}} \partial_{x_{i}} N(x-z) f(z) d z=\int_{0}^{x_{n}} \int_{\mathbb{R}^{n-1}} \partial_{x_{i} x_{j}}^{2} N(x-z) f(z) d z .
$$

Proof. The proof is given in Appendix B.

Next, we define the Hardy space $\mathscr{H}^{1}$. Let $\mathscr{N h}(x):=$ $\sup _{s>0}\left|h * \Gamma_{s}(x)\right|$. Let $\mathscr{H}^{1}\left(\mathbb{R}^{n}\right)$ be the space of functions $h$ so that $\mathscr{N} h \in L^{1}\left(\mathbb{R}^{n}\right)$ with the norm $\|h\|_{\mathscr{C}^{1}\left(\mathbb{R}^{n}\right)}:=$ $\|\mathscr{N} h\|_{L^{1}\left(\mathbb{R}^{n}\right)}$. Let $\mathscr{H}^{1}\left(\mathbb{R}_{+}^{n}\right)$ be the space of functions $h$ so that there is $\tilde{h} \in \mathscr{H}^{1}\left(\mathbb{R}^{n}\right)$ with $\left.\tilde{h}\right|_{\mathbb{R}_{+}^{n}}=h$ with the norm $\|h\|_{\mathscr{H}^{1}\left(\mathbb{R}_{+}^{n}\right)}$ $=\inf \left\{\|\tilde{h}\|_{\mathscr{H}^{1}\left(\mathbb{R}^{n}\right)}:\left.\tilde{h}\right|_{\mathbb{R}_{+}^{n}}=h\right\}$.

Next, we state a few well-known results related to the Hardy-norm estimates of the Gaussian kernel $\Gamma_{t}$

Proposition 4. Fix $a \in \mathbb{R}^{n}$. Then $1_{\mathbb{R}_{+}^{n}} D^{j}\left(\tau_{a} \Gamma_{t}\right) \in \mathscr{H}^{1}\left(\mathbb{R}^{n}\right)$ with

$$
\left\|1_{\mathbb{R}_{+}^{n}} D^{j}\left(\tau_{a} \Gamma_{t}\right)\right\|_{\mathscr{C}^{1}\left(\mathbb{R}^{n}\right)} \leq C t^{-j / 2}
$$

and

$$
\left\|1_{\mathbb{R}_{+}^{n}} D^{j}\left(\tau_{a} * \Gamma_{t}-\tau_{b} * \Gamma_{t}\right)\right\|_{\mathscr{H}^{1}\left(\mathbb{R}^{n}\right)} \leq C t^{-(j+\alpha) / 2} e^{-a_{n}{ }^{2} / 4 t}|\bar{a}-\bar{b}|^{\alpha}
$$

for $0<\alpha<1$.

We omit the proofs of well-known results of Proposition 4 . 
Proposition 5. Let $j=1 \cdots, n-1$ and $i=1, \cdots n$. Then we have

$$
\begin{aligned}
& \int_{\mathbb{R}_{+}^{n}}\left|\int_{0}^{x_{n}} \int_{\mathbb{R}^{n-1}} \frac{\partial^{2}}{\partial z_{i} \partial z_{j}} N(x-z) f(z, y) d z\right| d y \\
& \quad \leq C \sup _{x \in \mathbb{R}_{+}^{n}} \int_{\mathbb{R}_{+}^{n}}|f(x, y)| d y \\
& \quad+C x_{n}^{\alpha} \sup _{x_{n} / 2<z_{n}<x_{n}, \bar{z} \in \mathbb{R}^{n-1}} \frac{\int_{\mathbb{R}_{+}^{n}}\left|f\left(\bar{z}, z_{n}, y\right)-f\left(\bar{x}, z_{n}, y\right)\right| d y}{|\bar{x}-\bar{z}|^{\alpha}} .
\end{aligned}
$$

Proof. The proof is given in Appendix C.

\section{Estimate of $D^{j} e^{-A t} \mathbb{P} \nabla \cdot g$}

Solonikov in [9] and Shimizu in [16] provide the following estimates:

$$
\begin{gathered}
\left|\mathscr{D}^{j} e^{-A t} f\right|_{\infty} \leq C t^{-j / 2}|f|_{\infty}, \\
\left|e^{-A t} \mathbb{P} \nabla \cdot g\right|_{\infty} \leq C t^{-1 / 2}|g|_{\infty},
\end{gathered}
$$

where $f \in L^{\infty}\left(\mathbb{R}_{+}^{n}\right), g=\left(g_{i j}\right), 1 \leq i, j \leq n$, and $g_{i j} \in L^{\infty}\left(\mathbb{R}_{+}^{n}\right)$ for each $i, j$. Also, $f$ and $g$ vanish on the boundary. In addition, in paper [8] by Bae and Jin, they prove

$$
\left|\nabla e^{-A t} \mathbb{P} \nabla \cdot g\right|_{\infty} \leq C t^{-1 / 2}|\nabla g|_{\infty}
$$

as a critical estimate to prove their desired result.

With all the above estimates in hand, we begin to obtain the uniform estimate on the composite operator $D^{j} e^{-A t} \mathbb{P} \nabla \cdot g$. For that purpose, recall

$$
e^{-A t} f=\int_{\mathbb{R}_{+}^{n}} G(x, y, t) \cdot f(y) d y
$$

where $G(x, y, t)$ is defined by (8). In the following, consider $i \neq n$, and denote by $H=\left(H_{i j k}\right)_{i, j, k=1}^{n}$ the kernel tensor of the operator $e^{-A t} \mathbb{P} \nabla$ div. For simplicity in computational purpose, we consider $g_{i j}$ as a Schwartz class function in $\mathbb{R}_{+}^{n}$ vanishing on the boundary for each $i, j$. Thus, we begin by writing

$$
\left[\partial_{x_{i}} e^{-A t} \mathbb{P} D_{\bar{x}}^{\beta} \nabla \cdot g\right]_{i}(x)=\sum_{j, k=1}^{n} \int_{\mathbb{R}_{+}^{n}} \partial_{x_{i}} H_{i j k}(x, y, t) D_{\bar{y}}^{\beta} g_{j k}(y) d y,
$$

where

$$
\begin{aligned}
& H_{i j k}(x, y, t)=-\partial_{y_{k}} G_{i j}(x, y, t) \\
& \quad+\sum_{l=1}^{n} \partial_{y_{j}} \int_{\mathbb{R}_{+}^{n}} \partial_{y_{k}} \mathscr{G}(z, y) \partial_{z_{l}} G_{i l}(x, z, t) d z .
\end{aligned}
$$

With integration by parts, we obtain

$$
\left[\partial_{x_{i}} e^{-A t} \mathbb{P} D_{\bar{x}}^{\beta} \nabla \cdot g\right]_{i}(x)=\sum_{j, k=1}^{n}(-1)^{|\beta|} \int_{\mathbb{R}_{+}^{n}} \partial_{x_{i}} D_{\bar{y}}^{\beta} H_{i j k}(x, y, t) g_{j k}(y) d y .
$$

Use $G_{\text {in }}=0$ for $i \neq n$ to write the following:

$$
\begin{aligned}
\partial_{x_{i}} D_{\bar{y}}^{\beta} H_{i j k}(x, y, t) & =-\partial_{x_{i}} \partial_{y_{k}} D_{\bar{y}}^{\beta} G_{i j}(x, y, t) \\
& +\sum_{l=1}^{n-1} \partial_{x_{i}} \partial_{y_{j}} D_{\bar{y}}^{\beta} \int_{\mathbb{R}_{+}^{n}} \partial_{y_{k}} \mathscr{G}(z, y) \partial_{z_{l}} G_{i l}(x, z, t) d z .
\end{aligned}
$$

Use $\partial_{x_{i}} G_{i j}(x, y, t)=-\partial_{y_{i}} G_{i j}(x, y, t)$ for $i \neq n$ and Proposition 5 to justify the following expression:

$$
\begin{aligned}
\partial_{x_{i}} D_{\bar{y}}^{\beta} H_{i j k}(x, y, t)=\partial_{y_{k}} D_{\bar{y}}^{\beta+1} G_{i j}(x, y, t) \\
+\sum_{l=1}^{n-1} \partial_{x_{i}} \partial_{y_{j}} \int_{\mathbb{R}_{+}^{n}} \partial_{y_{k}} \mathscr{G}(z, y) \partial_{z_{l}} D_{\bar{z}}^{\beta} G_{i l}(x, z, t) \\
\cdot d z=\partial_{y_{k}} D_{\bar{y}}^{\beta+1} G_{i j}(x, y, t) \\
+\sum_{l=1}^{n-1} \partial_{y_{j}} \int_{\mathbb{R}_{+}^{n}} \partial_{y_{k}} \mathscr{G}(z, y) \partial_{x_{i}} D_{\bar{z}}^{\beta+1} G_{i l}(x, z, t) d z .
\end{aligned}
$$

Therefore, (29) can be rewritten as

$$
\begin{aligned}
& {\left[\partial_{x_{i}} e^{-A t} \mathbb{P} D_{\bar{x}}^{\beta} \nabla \cdot g\right]_{i}(x)=\sum_{j, k=1}^{n}(-1)^{|\beta|} \int_{\mathbb{R}_{+}^{n}}} \\
& \cdot\left[\partial_{y_{k}} D_{\bar{y}}^{\beta+1} G_{i j}(x, y, t)+\sum_{l=1}^{n-1} \partial_{y_{j}} \int_{\mathbb{R}_{+}^{n}} \partial_{y_{k}} \mathscr{G}(z, y) \partial_{x_{i}} D_{\bar{z}}^{\beta+1} G_{i l}(x, z, t) d z\right] \\
& \cdot g_{j k}(y) d y=I_{1}(x, t)+I_{2}(x, t),
\end{aligned}
$$

where

$$
\begin{aligned}
I_{1}(x, t):=(-1)^{|\beta|} \sum_{j, k=1}^{n} \int_{\mathbb{R}_{+}^{n}} \partial_{y_{k}} D_{\bar{y}}^{\beta+1} G_{i j}(x, y, t) g_{j k}(y) d y, \\
I_{2}(x, t):=(-1)^{|\beta|} \sum_{j, k=1}^{n} \int_{\mathbb{R}_{+}^{n}} \sum_{l=1}^{n-1} \partial_{y_{j}} \int_{\mathbb{R}_{+}^{n}} \partial_{y_{k}} \mathscr{G}(z, y) \partial_{x_{i}} \\
\cdot D_{\bar{z}}^{\beta+1} G_{i l}(x, z, t) d z g_{j k}(y) d y .
\end{aligned}
$$

First, we estimate $I_{1}$ for $k \neq n$. For that purpose, recall $\partial_{y_{k}} G_{i j}(x, y, t)=-\partial_{x_{k}} G_{i j}(x, y, t)$. Therefore,

$$
I_{1}(x, t)=\sum_{j, k=1, k \neq n}^{n} \int_{\mathbb{R}_{+}^{n}} D_{\bar{x}}^{\beta+2} G_{i j}(x, y, t) g_{j k}(y) d y .
$$

Clearly, $I_{1}(x, t)$ is the derivative of the $i$ th component of the solution of the Stokes equations. So, using estimate 
(23), we get the desired estimate on $I_{1}$ as below

$$
\left|I_{1}(x, t)\right| \leq C t^{-(j+1) / 2}|g|_{\infty} \quad \text { for } \quad k \neq n .
$$

Next, we estimate $I_{1}$ for $k=n$. In [9], $G_{i j}$ is given as

$$
G_{i j}(x, y, t)=\delta_{i j} \Gamma(x-y, t)+G_{i j}^{*}(x, y, t)
$$

where

$$
\begin{aligned}
& G_{i j}^{*}(x, y, t)=-\delta_{i j} \Gamma\left(x-y^{*}, t\right) \\
& \quad+4\left(1-\delta_{j n}\right) \partial_{x_{j}} \int_{0}^{x_{n}} \int_{\mathbb{R}^{n-1}} \partial_{x_{i}} N(x-z) \Gamma\left(z-y^{*}, t\right) d z .
\end{aligned}
$$

Next, we use the estimate

$$
\begin{gathered}
\left|D_{t}^{s} D_{x}^{k} D_{y}^{m} G_{i j}^{*}(x, y, t)\right| \leq C t^{-s-m_{n} / 2}\left(t+x_{n}^{2}\right)^{-k_{n} / 2} \\
\cdot\left(\left|x-y^{*}\right|^{2}+t\right)^{-\frac{n+\left|k^{\prime}\right|+\left|m^{\prime}\right|}{2}} e^{-\frac{C y_{n}^{2}}{t}}
\end{gathered}
$$

where $m^{\prime}=\left(m_{1}, m_{2}, \cdots m_{n-1}\right), k^{\prime}=\left(k_{1}, k_{2}, \cdots k_{n-1}\right)$, provided in [18], to obtain the desired estimate on $I_{1}$ for $k=n$.

Therefore, we use modified $G_{i j}$ for $k=n$ and rewrite $I_{1}$ as

$$
\begin{aligned}
I_{1}(x, t)= & (-1)^{|\beta|} \sum_{j, k=1}^{n} \int_{\mathbb{R}_{+}^{n}} \partial_{y_{n}} D_{\bar{y}}^{\beta+1}\left[\delta_{i j} \Gamma(x-y, t)\right. \\
& \left.+G_{i j}^{*}(x, y, t)\right] g_{j k}(y) d y \\
= & (-1)^{2|\beta|+1} \sum_{j, k=1}^{n} \int_{\mathbb{R}_{+}^{n}} \partial_{y_{n}} D_{\bar{x}}^{\beta+1} \delta_{i j} \Gamma(x-y, t) g_{j k} \\
& \cdot(y) d y+(-1)^{|\beta|} \sum_{j, k=1}^{n} \int_{\mathbb{R}_{+}^{n}} \partial_{y_{n}} D_{\bar{y}}^{\beta+1} G_{i j}^{*}(x, y, t) \\
& \cdot g_{j k}(y) d y:=I_{1}^{*}+I_{1}^{* *} .
\end{aligned}
$$

The estimate for $I_{1}^{*}$ follows as

$$
\begin{aligned}
& \left|I_{1}^{*}(x, t)\right| \leq C|g|_{\infty} \int_{\mathbb{R}^{n}}\left|1_{\mathbb{R}_{+}^{n}} \partial_{y_{n}} D_{\bar{x}}^{\beta+1} \delta_{i j} \Gamma(x-y, t)\right|
\end{aligned}
$$

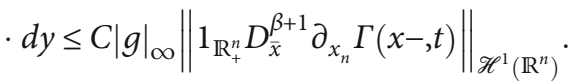

Applying Proposition 4, we obtain

$$
\left|I_{1}^{*}(x, t)\right| \leq C|g|_{\infty} t^{-(j+1) / 2}
$$

To estimate $I_{1}^{* *}$, we use the estimate of (38) and obtain

$$
\begin{gathered}
\left|I_{1}^{* *}(x, t)\right| \leq C|g|_{\infty} t^{-1 / 2} \int_{\mathbb{R}_{+}^{n}} \frac{e^{-C y_{n}^{2} / t}}{\left(\left|x-y^{*}\right|^{2}+t\right)^{n+j / 2}} d y \leq C|g|_{\infty} t^{-(j+1) / 2} \\
\cdot \int_{\mathbb{R}_{+}^{n}} \frac{e^{-C y_{n}^{2} / t}}{\left(\left|x-y^{*}\right|^{2}+t\right)^{n / 2}} d y \leq C|g|_{\infty} t^{-(j+1) / 2} t^{1 / 2}\left(t+x_{n}^{2}\right)^{-1 / 2} .
\end{gathered}
$$

Finally, we get

$$
\left|I_{1}^{* *}(x, t)\right| \leq C t^{-(j+1) / 2}|g|_{\infty} .
$$

We obtain

$$
\left|I_{1}\right|_{\infty} \leq\left|I_{1}^{*}\right|_{\infty}+\left|I_{1}^{* *}\right|_{\infty} \leq C t^{-(j+1) / 2}|g|_{\infty} \quad \text { for } \quad k=n \text {. }
$$

Therefore, from (35) and (44), we obtain

$$
\left|I_{1}\right|_{\infty} \leq C t^{-(j+1) / 2}|g|_{\infty} \text { for all } k
$$

Next, we estimate $I_{2}$ : For that, let us begin by rewriting $I_{2}$ after dropping the summation notations and negative signs for convenience in writing.

$$
I_{2}(x, t)=\int_{\mathbb{R}_{+}^{n}} \partial_{y_{j}} \int_{\mathbb{R}_{+}^{n}} \partial_{y_{k}} \mathscr{G}(z, y) \partial_{x_{i}} D_{\bar{z}}^{\beta+1} G_{i l}(x, z, t) d z g_{j k}(y) d y .
$$

Equivalently, we write

$$
I_{2}(x, t)=\int_{\mathbb{R}_{+}^{n}} T(x, y, t) g_{j k}(y) d y,
$$

where

$$
T(x, y, t):=\partial_{y_{j}} \int_{\mathbb{R}_{+}^{n}} \partial_{y_{k}} \mathscr{G}(z, y) D_{\bar{z}}^{\beta+2} G_{i l}(x, z, t) d z .
$$

Using expression for $G_{i j}$ from (8) for $i, l \neq n$, we obtain

$$
\begin{aligned}
T(x, y, t)= & \partial_{y_{j}} \int_{\mathbb{R}_{+}^{n}} \partial_{y_{k}} \mathscr{G}(z, y) \\
& \cdot D_{\bar{z}}^{\beta+2}\left[\delta_{i l}\left[\Gamma(x-z, t)-\Gamma\left(x-z^{*}, t\right)\right]\right. \\
& \left.+4 \partial_{x_{l}} \int_{0}^{x_{n}} \int_{\mathbb{R}^{n-1}} \partial_{x_{i}} N(x-w) \Gamma\left(w-z^{*}, t\right) d w\right] \\
& \cdot d z=T_{1}+T_{2}+T_{3},
\end{aligned}
$$

where

$$
\begin{aligned}
& T_{1}(x, y, t):=\partial_{y_{j}} \int_{\mathbb{R}_{+}^{n}} \partial_{y_{k}} \mathscr{G}(z, y) D_{\bar{z}}^{\beta+2} \delta_{i l} \Gamma(x-z, t) d z, \\
& T_{2}(x, y, t):=-\partial_{y_{j}} \int_{\mathbb{R}_{+}^{n}} \partial_{y_{k}} \mathscr{G}(z, y) D_{\bar{z}}^{\beta+2} \delta_{i l} \Gamma\left(x-z^{*}, t\right) d z, \\
& T_{3}(x, y, t):=\partial_{y_{j}} \int_{\mathbb{R}_{+}^{n}} \partial_{y_{k}} \mathscr{G}(z, y) \\
& \cdot D_{\bar{z}}^{\beta+2}\left[4 \partial_{x_{l}} \int_{0}^{x_{n}} \int_{\mathbb{R}^{n-1}} \partial_{x_{i}} N(x-w) \Gamma\left(w-z^{*}, t\right) d w\right] d z .
\end{aligned}
$$


To estimate $T_{1}$, let us proceed by writing

$$
\begin{aligned}
& T_{1}(x, y, t)=\partial_{y_{j}} \int_{\mathbb{R}_{+}^{n}} \partial_{y_{k}}\left[N(z-y)+N\left(z-y^{*}\right)\right] \\
& \cdot D_{\bar{z}}^{\beta+2} \delta_{i l} \Gamma(x-z, t) d z=\partial_{y_{j}} \int_{\mathbb{R}^{n}} \partial_{y_{k}} N(z-y) 1_{\left\{z_{n}>0\right\}} \\
& \cdot D_{\bar{z}}^{\beta+2} \delta_{i l} \Gamma(x-z, t) d z+\partial_{y_{j}} \int_{\mathbb{R}^{n}} \partial_{y_{k}} N(z-y) 1_{\left\{z_{n}<0\right\}} \\
& \cdot D_{\bar{z}}^{\beta+2} \delta_{i l} \Gamma\left(x-z^{*}, t\right) d z .
\end{aligned}
$$

It is well known that $1_{\mathbb{R}_{+}^{n}} D_{\bar{z}}^{\beta+2} \Gamma(x-z, t)$ and $1_{\mathbb{R}_{-}^{n}} D_{\bar{z}}^{\beta+2} \Gamma($ $\left.x-z^{*}, t\right)$ are in Hardy space $\mathscr{H}^{1}\left(\mathbb{R}^{n}\right)$, for any fixed $z \in \mathbb{R}^{n}$. Since the Calderon-Zygmund type transforms are bounded in Hardy space, we obtain that

$$
\begin{aligned}
& \int_{\mathbb{R}_{+}^{n}}\left|T_{1}(x, y, t)\right| d y \leq C\left\|1_{\mathbb{R}_{+}^{n}} D_{\bar{z}}^{\beta+2} \Gamma_{t}(z-\cdot)\right\|_{\mathscr{H}^{1}\left(\mathbb{R}^{n}\right)} \\
& +C\left\|1_{\mathbb{R}_{-}^{n}} D_{\bar{z}}^{\beta+2} \Gamma_{t}(z-\cdot)\right\|_{\mathscr{H}^{1}\left(\mathbb{R}^{n}\right)} .
\end{aligned}
$$

Using the estimates of Proposition 4, we arrive at

$$
\int_{\mathbb{R}_{+}^{n}}\left|T_{1}(x, y, t)\right| d y \leq C t^{-(j+1) / 2}
$$

With exactly the same argument as for $T_{1}$, we also obtain

$$
\int_{\mathbb{R}_{+}^{n}}\left|T_{2}(x, y, t)\right| d y \leq C t^{-(j+1) / 2} .
$$

It remains to obtain an estimate for $T_{3}$. We use Proposition 3 for $i, l \neq n$ by replacing $f$ by $\Gamma(w,$.$) and also use G_{\ln }=0$ to rewrite $T_{3}$ as

$$
\begin{aligned}
& T_{3}(x, y, t)=4 \partial_{y_{j}} \int_{\mathbb{R}_{+}^{n}} \partial_{y_{k}} \mathscr{G}(z, y) \\
& \cdot D_{\bar{z}}^{\beta+2}\left[-\frac{\delta_{i l}}{2 n} \Gamma\left(x-z^{*}, t\right)+\int_{0}^{x_{n}} \int_{\mathbb{R}^{n-1}} \partial_{x_{i} x_{l}}^{2} N(x-w)\right. \\
& \left.\cdot \Gamma\left(w-z^{*}, t\right) d w\right] d z=-\frac{\delta_{i l}}{2 n} \partial_{y_{j}} \int_{\mathbb{R}_{+}^{n}} \partial_{y_{k}} \mathscr{G}(z, y) \\
& \cdot D_{\bar{z}}^{\beta+2} \Gamma\left(x-z^{*}, t\right) d z+\partial_{y_{j}} \int_{\mathbb{R}_{+}^{n}} \partial_{y_{k}} \mathscr{G}(z, y) \int_{0}^{x_{n}} \int_{\mathbb{R}^{n-1}} \\
& \cdot \partial_{x_{i} x_{l}}^{2} N(x-w) D_{\bar{z}}^{\beta+2} \Gamma\left(w-z^{*}, t\right) d w d z:=T_{3}^{*}+T_{3}^{* *} .
\end{aligned}
$$

By the same argument as for $T_{1}$, we can obtain

$$
\int_{\mathbb{R}_{+}^{n}}\left|T_{3}^{*}(x, y, t)\right| d y \leq C t^{-(j+1) / 2} .
$$

Let us rewrite $T_{3}^{* *}$ as

$$
\begin{aligned}
T_{3}^{* *}(x, y, t)= & \int_{0}^{x_{n}} \int_{\mathbb{R}^{n-1}} \partial_{x_{i} x_{l}}^{2} N(x-w) \\
& \cdot\left[\partial_{y_{j}} \int_{\mathbb{R}_{+}^{n}} \partial_{y_{k}} \mathscr{G}(z, y) D_{\bar{z}}^{\beta+2} \Gamma\left(w-z^{*}, t\right) d z\right] d w .
\end{aligned}
$$

Set

$$
T_{j k}(w, y, t):=\partial_{y_{j}} \int_{\mathbb{R}_{+}^{n}} \partial_{y_{k}} \mathscr{G}(z, y) D_{\bar{z}}^{\beta+2} \Gamma\left(w-z^{*}, t\right) d z
$$

By Proposition 5,

$$
\begin{aligned}
& \int_{\mathbb{R}_{+}^{n}}\left|T_{3}^{* *}(x, y, t)\right| d y \leq C \sup _{w \in \mathbb{R}_{+}^{n}} \int_{\mathbb{R}_{+}^{n}}\left|T_{j k}(w, y, t)\right| d y \\
& +C x_{n}^{\alpha} \sup _{x_{n} / 2<w_{n}<x_{n}} \sup _{\bar{w} \in \mathbb{R}^{n-1}} \frac{\int_{\mathbb{R}_{+}^{n}}\left|T_{j k}\left(\left(\bar{w}, w_{n}\right), y, t\right)-T_{j k}\left(\left(\bar{x}, w_{n}\right), y, t\right)\right| d y}{|\bar{x}-\bar{w}|^{\alpha}} .
\end{aligned}
$$

Notice that

$$
\begin{aligned}
& T_{j k}(w, y, t)=\partial_{y_{j}} \int_{\mathbb{R}_{+}^{n}} \partial_{y_{k}}\left[N(z-y)+N\left(z-y^{*}\right)\right] \\
& \cdot D_{\bar{z}}^{\beta+2} \Gamma\left(w-z^{*}, t\right) d z=\partial_{y_{j}} \int_{\mathbb{R}^{n}} \partial_{y_{k}} N(z-y) 1_{\left\{z_{n}>0\right\}} \\
& \cdot D_{\bar{z}}^{\beta+2} \Gamma\left(w-z^{*}, t\right) d z+\partial_{y_{j}} \int_{\mathbb{R}^{n}} \partial_{y_{k}} N(z-y) 1_{\left\{z_{n}<0\right\}} \\
& \cdot D_{\bar{z}}^{\beta+2} \Gamma(w-z, t) d z .
\end{aligned}
$$

We also recall that $1_{\mathbb{R}_{+}^{n}} D_{w}^{\beta+2} \Gamma\left(w-z^{*}, t\right)$ and $1_{\mathbb{R}_{-}^{n}} D_{w}^{\beta+2} \Gamma($ $w-z, t)$ are in the Hardy space $\mathscr{H}^{1}\left(\mathbb{R}^{n}\right)$, for any fixed $w \in$ $\mathbb{R}^{n}$. Since the Calderon-Zygmund type transforms are bounded in Hardy space, after using $D_{\bar{w}}^{\beta+2} \Gamma(w-z, t)=$ $(-1)^{|\beta|+2} D_{\bar{z}}^{\beta+2} \Gamma(w-z, t)$, we arrive at

$$
\begin{gathered}
\int_{\mathbb{R}_{+}^{n}}\left|T_{j k}(w, y, t)\right| d y \leq C\left\|1_{\mathbb{R}_{+}^{n}} D_{\bar{w}}^{\beta+2} \Gamma(w-\cdot, t)\right\|_{\mathscr{H}^{1}\left(\mathbb{R}^{n}\right)} \\
+\left\|1_{\mathbb{R}_{-}^{n}} D_{\bar{w}}^{\beta+2} \Gamma(w-\cdot, t)\right\|_{\mathscr{H}^{1}\left(\mathbb{R}^{n}\right)} \leq C t^{-(j+1) / 2} .
\end{gathered}
$$

Let us recall a result of Proposition 4:

$$
1_{\mathbb{R}_{+}^{n}} D_{w}^{\beta+2}\left[\left(\tau_{a} * \Gamma_{t}\right)-\left(\tau_{b} * \Gamma_{t}\right)\right] \in \mathscr{H}^{1}\left(\mathbb{R}^{n}\right),
$$

for any $a, b \in \mathbb{R}^{n}$, and is bounded by $C t^{-(|\beta|+2+\gamma) / 2} e^{-w_{n}^{2} / 4 t}$ $|\bar{w}-\bar{x}|^{\gamma}$ for $0<\gamma<1$. Hence, in similar way as for $P_{j k}$, we obtain

$\int_{\mathbb{R}_{+}^{n}}\left|P_{j k}\left(\left(\bar{w}, w_{n}\right), y, t\right)-P_{j k}\left(\left(\bar{x}, w_{n}\right), y, t\right)\right| d y \leq C t^{-(|\beta|+2+\gamma) / 2} e^{\frac{-w_{n}^{2}}{4 t}}|\bar{w}-\bar{x}|^{\gamma}$. 
Therefore,

$$
\int_{\mathbb{R}_{+}^{n}}\left|T_{3}^{* *}(x, y, t)\right| d y \leq C t^{-(|\beta|+2) / 2}+C t^{-(|\beta|+2+\gamma) / 2} x_{n}^{\gamma} e^{\frac{-x_{n}^{2}}{4 t}} \leq C t^{-(j+1) / 2} .
$$

Using (56) and (64) leads us to obtain

$$
\int_{\mathbb{R}_{+}^{n}}\left|T_{3}(x, y, t)\right| d y \leq C t^{-(j+1) / 2} .
$$

Since $T=T_{1}+T_{2}+T_{3}$, with the use of (53), (54), and (65), we obtain

$$
\left|I_{2}(x, t)\right| \leq|g|_{\infty} \int_{\mathbb{R}_{+}^{n}}|T(x, y, t)| d y \leq C t^{-(j+1) / 2}|g|_{\infty} .
$$

Finally, using (45) and (66) with fact that $e^{-A t}$ commutes with $D_{\bar{x}}^{\beta}$, we have proved the following important lemma.

Lemma 6. For any $g=(g)_{i j}, 1 \leq i, j \leq n$ with $g_{i j} \in L^{\infty}\left(\mathbb{R}_{+}^{n}\right)$, and $g_{i j}(\bar{x}, 0)=0$, there exists a constant $C$ independent of $t$ and $g$ such that

$$
\left|D^{j} e^{-A t} \mathbb{P}(\nabla \cdot g)\right|_{\infty} \leq C t^{-(j+1) / 2}|g|_{\infty},
$$

for $0<t<T$, for some $T>0$.

Corollary 7. Let $g$ be as in the previous lemma, then the solution of

$$
u_{t}+A u=\mathbb{P} \nabla \cdot g,\left.\quad u\right|_{t=0}=0,\left.\quad u\right|_{x_{n}=0}=0
$$

satisfies

$$
|u(t)|_{\infty} \leq C t^{1 / 2} \max _{0 \leq s \leq t}|g(s)|_{\infty}, \quad 0<t<T,
$$

for some $T>0$.

Proof. The solution of (38) is given by

$$
u(t)=\int_{0}^{t} e^{-A(t-s)} \mathbb{P} \nabla \cdot g(s) d s, \quad 0<t<T
$$

and

$$
|u(t)|_{\infty} \leq \int_{0}^{t}\left|e^{-A(t-s)} \mathbb{P} \nabla \cdot g(s)\right|_{\infty} d s
$$

Applying the estimate (24), we obtain

$$
|u(t)|_{\infty} \leq \max _{0 \leq s \leq t}|g(s)|_{\infty} \int_{0}^{t}(t-s)^{-1 / 2} d s .
$$

Hence, we obtain

$$
|u(t)|_{\infty} \leq C t^{1 / 2} \max _{0 \leq s \leq t}|g(s)|_{\infty} .
$$

\section{Estimates for the Navier-Stokes Equations}

Recall the transformed abstract ordinary differential equation (3):

$$
u_{t}+A u=-\mathbb{P}(u \cdot \nabla u)
$$

Solution of (74) with given initial and boundary condition as in (1) is given by

$$
u(t)=e^{-A t} f-\int_{0}^{t} e^{-A(t-s)} \mathbb{P}(u \cdot \nabla u)(s) d s .
$$

Using the solution (75) along with the use of estimates (23), (24), and (25), we prove the following important lemma.

Lemma 8. Set

$$
V(t)=|u(t)|_{\infty}+t^{1 / 2}|\mathscr{D} u(t)|_{\infty}, \quad 0<t<T .
$$

There is a constant $C>0$, independent of $t$ and $f$, so that

$$
V(t) \leq C|f|_{\infty}+C t^{1 / 2} \max _{0 \leq s \leq t} V^{2}(t), \quad 0<t<T .
$$

Proof. Using estimate (23) for the solution of the Stokes equations in (75), we obtain

$$
|u(t)|_{\infty} \leq|f|_{\infty}+\left|\int_{0}^{t} e^{-A(t-s)}(\mathbb{P} \nabla \cdot g)(s) d s\right|_{\infty} .
$$

From (74), after using estimate (24), with the fact that $g$ is quadratic in $u$ gives us

$$
\begin{gathered}
|u(t)|_{\infty} \leq|f|_{\infty}+C \int_{0}^{t}(t-s)^{-1 / 2}|u(s)|_{\infty}^{2} d s=|f|_{\infty} \\
+C \int_{0}^{t}(t-s)^{-1 / 2} s^{-1 / 2} s^{1 / 2}|u(s)|_{\infty}^{2} d s \leq|f|_{\infty} \\
+C \max _{0 \leq s \leq t}\left\{s^{1 / 2}|u(s)|_{\infty}^{2}\right\} \int_{0}^{t}(t-s)^{-1 / 2} s^{-1 / 2} d s .
\end{gathered}
$$

Since $\int_{0}^{t}(t-s)^{-1 / 2} s^{-1 / 2} d s=C>0$, which is independent of $t$, we arrive at the following estimate

$$
|u(t)|_{\infty} \leq|f|_{\infty}+C \max _{0 \leq s \leq t}\left\{s^{1 / 2}|u(s)|_{\infty}^{2}\right\},
$$

$$
|u(t)|_{\infty} \leq|f|_{\infty}+C t^{1 / 2} \max _{0 \leq s \leq t} V^{2}(s) .
$$

Next, apply $D_{i}$ to $u(t)$ in the integral form to obtain and 
estimate for $D_{i} u=: v$ :

$$
\begin{aligned}
v(t) & =D_{i} e^{-A t} f-D_{i} \int_{0}^{t} e^{-A(t-s)} \mathbb{P} \nabla \cdot g(u(s)) d s=D_{i} e^{-A t} f \\
- & \int_{0}^{t} D_{i} e^{-A(t-s)} \mathbb{P} \nabla \cdot g(u(s)) d s .
\end{aligned}
$$
below.

Let us estimate the integral in the above expression as

$$
\left|\int_{0}^{t} D_{i} e^{-A(t-s)}(\mathbb{P} \nabla \cdot g)(s) d s\right| \leq \int_{0}^{t}\left|D_{i} e^{-A(t-s)}(\mathbb{P} \nabla \cdot g)(s)\right| d s .
$$

We use the estimate (25) again with the fact that $g$ is quadratic in $u$ to obtain

$$
\begin{gathered}
\left|\int_{0}^{t} D_{i} e^{-A(t-s)}(\mathbb{P} \nabla \cdot g)(s) d s\right| \leq C \int_{0}^{t}(t-s)^{-1 / 2}|u(s)|_{\infty}|\mathscr{D} u(s)|_{\infty} \\
\cdot d s=C \int_{0}^{t}(t-s)^{-1 / 2} s^{-1 / 2} s^{1 / 2}|u(s)|_{\infty}|\mathscr{D} u(s)|_{\infty} \\
\cdot d s \leq C \max _{0 \leq s \leq t}\left\{s^{1 / 2}|u(s)|_{\infty}|\mathscr{D u}(s)|_{\infty}\right\} \int_{0}^{t}(t-s)^{-1 / 2} s^{-1 / 2} \\
\cdot d s \leq C \max _{0 \leq s \leq t}\left\{|u(s)|_{\infty}^{2}+s|\mathscr{D u}(s)|_{\infty}^{2}\right\} .
\end{gathered}
$$

Therefore, we have the following estimate for $v=D_{i} u$

$$
\begin{gathered}
|v(t)|_{\infty} \leq C t^{-1 / 2}|f|_{\infty}+C \max _{0 \leq s \leq t}\left\{|u(s)|_{\infty}^{2}+s|\mathscr{D u}(s)|_{\infty}^{2}\right\} \\
t^{1 / 2}|\mathscr{D} u(t)|_{\infty} \leq C|f|_{\infty}+C t^{1 / 2} \max _{0 \leq s \leq t} V^{2}(t)
\end{gathered}
$$

The combination of (80) and (85), proves Lemma 8.

Lemma 9. Let $C$ and $u \in L^{\infty}\left(\mathbb{R}_{+}^{n} \times(0, T)\right)$ be same as in Lemma 8 for some $T>0$. Set

$$
c_{0}=\frac{1}{16 C^{4}}
$$

Then $T>c_{0} /|f|_{\infty}^{2}$ and

$$
|u(t)|_{\infty}+t^{1 / 2}|\mathscr{D u}(t)|_{\infty}<2 C|f|_{\infty} \quad \text { for } \quad 0 \leq t<\frac{c_{0}}{|f|_{\infty}^{2}}
$$

Proof. We prove this lemma by contradiction after recalling the definition of $V(t)$ in (76). Suppose that (88) does not hold, then denote by $t_{0}$ the smallest time with $V\left(t_{0}\right)=2 C$ $|f|_{\infty}$. Use (77) to obtain

$$
2 C|f|_{\infty}=V\left(t_{0}\right) \leq C|f|_{\infty}+C t_{0}^{1 / 2} 4 C^{2}|f|_{\infty}^{2} .
$$

Thus

$$
1 \leq 4 C^{2} t_{0}^{1 / 2}|f|_{\infty}^{2}
$$

Therefore, $t_{0} \geq c_{0} /|f|_{\infty}^{2}$. This contradiction proves (88) and $T>c_{0} /|f|_{\infty}^{2}$.

\section{Proof of Theorem 1}

Lemma 9 proves Theorem 1 for $j=0,1$ for $0<t<c_{0} /|f|_{\infty}^{2}$. Now, we apply induction on $j$ to prove Theorem 1. Suppose $j \geq 1$ and assume

$$
t^{k / 2}\left|\mathscr{D}^{k} u(t)\right|_{\infty} \leq K_{k}|f|_{\infty}, \quad \text { for } \quad 0 \leq t \leq \frac{c_{0}}{|f|_{\infty}^{2}}, \quad 0 \leq k \leq j-1 .
$$

Apply $D^{\beta}:=D_{\bar{x}}^{\beta}$ to $u_{t}+A u=-\mathbb{P} \nabla \cdot g$ with the fact that $\mathbb{P}$ commutes with $D^{\beta}$. Also let $D^{\beta} u:=v$ to obtain

$$
v_{t}+A v=-D^{\beta} \mathbb{P} \nabla \cdot g \quad \text { with }\left.\quad v\right|_{\partial \mathbb{R}_{+}^{n}}=0, \quad \nabla \cdot v=0 .
$$

The solution of above system can be written as

$$
v(t)=D^{\beta} e^{-A t} f-\int_{0}^{t} e^{-A(t-s)} D^{\beta} \mathbb{P} \nabla \cdot g(u)(s) d s .
$$

Since $\nabla \cdot v=0$, we can write

$$
\partial_{x_{n}} v(t)=-\sum_{i=1}^{n-1} \partial_{x_{i}} v(t)
$$

Using integral form of $v(t)$ from above, we can write

$$
\partial_{x_{n}} v(t)=-\sum_{i=1}^{n-1} \partial_{x_{i}}\left[D^{\beta} e^{-A t} f-\int_{0}^{t} e^{-A(t-s)} D^{\beta} \mathbb{P} \nabla \cdot g(u)(s)\right] d s .
$$

Our goal is to prove $\left|\partial_{x_{n}} v(t)\right|_{\infty} \leq C t^{-j / 2}|f|_{\infty}$. For that, let us start with the following where $i \neq n$.

$$
\left|\partial_{x_{n}} v(t)\right|_{\infty} \leq C\left|D^{\beta+1} e^{-A t} f\right|_{\infty}+C\left|\partial_{x_{i}} \int_{0}^{t} e^{-A(t-s)} D^{\beta} \mathbb{P} \nabla \cdot g(u)(s) d s\right|_{\infty} .
$$

Using the estimate (23) in the first term of the above expression, we obtain

$$
\begin{aligned}
& \left|\partial_{x_{n}} v(t)\right|_{\infty} \leq C t^{-\mathrm{j} / 2} \mid \\
& \left.\cdot f\right|_{\infty}+C\left|\partial_{x_{i}} \int_{0}^{t / 2} e^{-A(t-s)} D^{\beta} \mathbb{P} \nabla \cdot g(u)(s) d s\right|_{\infty}+C \mid \partial_{x_{i}} \int_{t / 2}^{t} e^{-A(t-s)} \infty \\
& \cdot D^{\beta} \mathbb{P} \nabla \cdot g(u)(s) d s \mid
\end{aligned}
$$




$$
\leq C t^{-j / 2}|f|_{\infty}+I_{1}+I_{2}
$$

where

$$
\begin{aligned}
& I_{1}(x, t):=C\left|\partial_{x_{i}} \int_{0}^{t / 2} e^{-A(t-s)} D^{\beta} \mathbb{P} \nabla \cdot g(u)(s) d s\right|_{\infty}, \\
& I_{2}(x, t):=C\left|\partial_{x_{i}} \int_{t / 2}^{t} e^{-A(t-s)} D^{\beta} \mathbb{P} \nabla \cdot g(u)(s) d s\right|_{\infty}
\end{aligned}
$$

To estimate $I_{1}$ uniformly, we proceed as

$$
\left|I_{1}\right|_{\infty} \leq C \int_{0}^{t / 2}\left|\partial_{x_{i}} e^{-A(t-s)} D^{\beta} \operatorname{PP} \cdot g(u)(s)\right|_{\infty} d s
$$

Using Lemma 6, we obtain

$$
\left|I_{1}\right|_{\infty} \leq C \int_{0}^{t / 2}(t-s)^{-(j+1) / 2}|g(u(s))|_{\infty} d s
$$

We use simple integration, and the fact that $g$ is quadratic in $u$ to arrive at

$$
\left|I_{1}\right|_{\infty} \leq C t^{(1-j) / 2}|f|_{\infty}^{2}
$$

Next, we estimate $I_{2}$. For that, we proceed in the following way:

$$
\left|I_{2}\right|_{\infty} \leq C \int_{t / 2}^{t}\left|\partial_{x_{i}} e^{-A(t-s)} D^{\beta} \operatorname{PP} \cdot g(u)(s)\right|_{\infty} d s
$$

Since the order of the derivatives of $\left|D^{\beta} \nabla \cdot g\right|_{\infty}$ is $|\beta|+1$, for convenience in writing, we use $\left|D^{j} g\right|_{\infty}$ to estimate $\left|D^{\beta} \nabla \cdot g\right|_{\infty}$. Since $g(u)$ is quadratic in $u$; therefore

$$
\left|D^{j} g(u)\right|_{\infty} \leq C|u|_{\infty}\left|\mathscr{D}^{j} u\right|_{\infty}+\sum_{k=1}^{j-1}\left|\mathscr{D}^{k} u\right|_{\infty}\left|\mathscr{D}^{j-k} u\right|_{\infty}
$$

By induction hypothesis (91) we obtain

$$
\sum_{k=1}^{j-1}\left|\mathscr{D}^{k} u(s)\right|_{\infty}\left|\mathscr{D}^{j-k} u(s)\right|_{\infty} \leq C s^{-j / 2}|f|_{\infty}^{2}
$$

Apply estimate (25) to the integral (103) with the use of (104) to obtain

$$
\begin{aligned}
& \left|I_{2}(t)\right|_{\infty} \leq C \int_{t / 2}^{t}(t-s)^{-1 / 2} \\
& \quad \cdot\left(C|u(s)|_{\infty}\left|\mathscr{D}^{j} u(s)\right|_{\infty}+\sum_{k=1}^{j-1}\left|\mathscr{D}^{k} u(s)\right|_{\infty}\left|\mathscr{D}^{j-k} u(s)\right|_{\infty}\right) d s=J_{1}+J_{2},
\end{aligned}
$$

where

$$
\begin{gathered}
J_{1}(x, t):=\left.C \int_{t / 2}^{t}(t-s)^{-1 / 2}\left|u(s)_{\infty}\right| \mathscr{D}^{j} u(s)\right|_{\infty} d s, \\
J_{2}(x, t):=C \sum_{k=1}^{j-1} \int_{t / 2}^{t}(t-s)^{-1 / 2}\left|\mathscr{D}^{k} u(s)\right|_{\infty}\left|\mathscr{D}^{j-k} u(s)\right|_{\infty} d s .
\end{gathered}
$$

Since $\int_{t / 2}^{t}(t-s)^{-1 / 2} s^{-j / 2} d s=C t^{(1-j) / 2}$, where $C$ is independent of $t$, and the using the estimate of (105), we obtain $\left|J_{2}(t)\right|_{\infty} \leq C|f|_{\infty}^{2} t^{(1-j) / 2}$. For $J_{1}$, let us begin as below.

$$
\begin{gathered}
\left|J_{1}(t)\right|_{\infty} \leq C \int_{t / 2}^{t}(t-s)^{-1 / 2}|u(s)|_{\infty}\left|\mathscr{D}^{j} u(s)\right|_{\infty} \\
\cdot d s \leq C|f|_{\infty} \int_{t / 2}^{t}(t-s)^{-1 / 2} s^{-j / 2} s^{j / 2}\left|\mathscr{D}^{j} u(s)\right|_{\infty} \\
\cdot d s \leq C|f|_{\infty} t^{(1-j) / 2} \max _{0 \leq s \leq t}\left\{\left.s^{j / 2} \mathscr{D}^{j} u(s)\right|_{\infty}\right\} .
\end{gathered}
$$

Therefore

$$
\begin{gathered}
\left|I_{2}(t)\right|_{\infty} \leq\left|J_{1}(t)\right|_{\infty}+\left|J_{2}(t)\right|_{\infty} \\
\left|I_{2}(t)\right|_{\infty} \leq C t^{(1-j) / 2}|f|_{\infty}^{2}+C|f|_{\infty} t^{(1-j) / 2} \max _{0 \leq s \leq t}\left\{\left.s^{j / 2} \mathscr{D}^{j} u(s)\right|_{\infty}\right\} .
\end{gathered}
$$

We use these bounds to bind the integral in (97). We have $D^{j} u=\partial_{x_{n}} D^{\beta} u$. Then, maximizing the resulting estimate for $t^{j / 2}\left|D^{j} u(t)\right|_{\infty}$ over all derivatives $D^{j}$ of order $j$ and setting

$$
\phi(t):=t^{j / 2}\left|\mathscr{D}^{j} u(t)\right|_{\infty}
$$

and from (98), we obtain the following estimate:

$$
\phi(t) \leq C|f|_{\infty}+C t^{1 / 2}|f|_{\infty}^{2}+C|f|_{\infty} t^{1 / 2} \max _{0 \leq s \leq t} \phi(s) \quad \text { for } \quad 0 \leq t \leq \frac{c_{0}}{|f|_{\infty}^{2}}
$$

Since $t^{1 / 2}|f|_{\infty} \leq \sqrt{c_{0}}$, then $C t^{1 / 2}|f|_{\infty}^{2} \leq C \sqrt{c_{0}}|f|_{\infty}$. Therefore

$$
\phi(t) \leq C_{j}|f|_{\infty}+C_{j}|f|_{\infty} t^{1 / 2} \max _{0 \leq s \leq t} \phi(s) \quad \text { for } \quad 0 \leq t \leq c_{0} /|f|_{\infty}^{2} .
$$

Let us fix $C_{j}$ so that the above estimate holds and set

$$
c_{j}=\min \left\{c_{0}, \frac{1}{4 C_{j}^{2}}\right\} .
$$


First, let us prove the following:

$$
\phi(t)<2 C_{j}|f|_{\infty} \quad \text { for } \quad 0 \leq t<\frac{c_{j}}{|f|_{\infty}^{2}}
$$

Suppose there is a smallest time $t_{0}$ such that $0<t_{0}<c_{j}$ l $|f|_{\infty}^{2}$ with $\phi\left(t_{0}\right)=2 C_{j}|f|_{\infty}$. Then, using (88), we obtain

$$
2 C_{j}|f|_{\infty}=\phi\left(t_{0}\right) \leq C_{j}|f|_{\infty}+2 C_{j}^{2}|f|_{\infty}^{2} t_{0}^{1 / 2}
$$

Thus

$$
1 \leq 2 C_{j}|f|_{\infty} t_{0}^{1 / 2} \quad \text { gives } \quad t_{0} \geq c_{j} /|f|_{\infty}^{2}
$$

which contradicts the assertion. Therefore, we proved the estimate

$$
t^{j / 2}\left|\mathscr{D}^{j} u(t)\right|_{\infty} \leq 2 C_{j}|f|_{\infty} \quad \text { for } \quad 0 \leq t \leq c_{j}|f|_{\infty}^{2}
$$

If

$$
T_{j}:=\frac{c_{j}}{|f|_{\infty}^{2}}<t \leq \frac{c_{0}}{|f|_{\infty}^{2}}=: T_{0}
$$

then we start the corresponding estimate at $t-T_{j}$. Using Lemma 9, we have $\left|u\left(t-T_{j}\right)\right|_{\infty} \leq 2|f|_{\infty}$ and obtain

$$
T_{j}^{j / 2}\left|\mathscr{D}^{j} u(t)\right|_{\infty} \leq 4 C_{j}|f|_{\infty}
$$

Finally, for any $t$ satisfying (118)

$$
t^{j / 2} \leq T_{0}^{j / 2}=\left(\frac{c_{0}}{c_{j}}\right)^{j / 2} T_{j}^{j / 2},
$$

and (119) yield

$$
t^{j / 2}\left|\mathscr{D}^{j} u(t)\right|_{\infty} \leq 4 C_{j}\left(\frac{c_{0}}{c_{j}}\right)^{j / 2}|f|_{\infty} .
$$

This completes the proof of Theorem 1.

In the following appendices, we provide proofs of the propositions that are introduced in "Some Auxiliary Results." However, these proofs have also been provided in [8]. For the reader's convenience, we provide them with more details in this paper as well.

\section{Appendix}

\section{A. Proof of Proposition 2}

We first let the case $k \neq n$. Differentiate $G_{i j}$ with respect to $x_{k}$ to obain

$$
\begin{gathered}
\partial_{x_{k}} G_{i j}(x, y, t)=\delta_{i j} \partial_{x_{k}}\left[\Gamma_{t}(x-y)-\Gamma_{t}\left(x-y^{*}\right)\right] \\
+4\left(1-\delta_{j n}\right) \partial_{x_{k}} \partial_{x_{j}} \int_{0}^{x_{n}} \int_{\mathbb{R}^{n-1}} \partial_{x_{i}} N(x-z) \Gamma_{t}\left(z-y^{*}\right) \\
\cdot d z=\delta_{i j} \partial_{x_{k}}\left[\Gamma_{t}(x-y)-\Gamma_{t}\left(x-y^{*}\right)\right]+4\left(1-\delta_{j n}\right) \\
\cdot \partial_{x_{j}} \int_{0}^{x_{n}} \int_{\mathbb{R}^{n-1}} \partial_{x_{i}} N(x-z) \partial_{z_{k}} \Gamma_{t}\left(z-y^{*}\right) d z .
\end{gathered}
$$

Observe that for $k \neq n$

$$
\begin{gathered}
\partial_{y_{k}} \Gamma_{t}(x-y)=-\partial_{x_{k}} \Gamma_{t}(x-y), \\
\partial_{y_{k}} \Gamma_{t}\left(x-y^{*}\right)=-\partial_{x_{k}} \Gamma_{t}\left(x-y^{*}\right) .
\end{gathered}
$$

This proves the desired result of Proposition 2 for $k \neq n$. For the case $k=n$. We start with the expression for some appropriately chosen function $g$ :

$$
\begin{gathered}
\partial_{x_{n}} \int_{0}^{x_{n}} \int_{\mathbb{R}^{n-1}} \partial_{x_{i}} N(x-z) g(z) d z=\int_{0}^{x_{n}} \int_{\mathbb{R}^{n-1}} \partial_{x_{i}} \partial_{x_{n}} N(x-z) g(z) \\
\cdot d z+\frac{\delta_{\text {in }}}{2 n} g(x)+\int_{\mathbb{R}^{n-1}} \partial_{x_{i}} N(\bar{x}-\bar{z}, 0) g\left(\bar{z}, x_{n}\right) d \bar{z},
\end{gathered}
$$

and

$$
\begin{aligned}
& -\int_{0}^{x_{n}} \int_{\mathbb{R}^{n-1}} \partial_{x_{i}} \partial_{x_{n}} N(x-z) g(z) d z=\int_{0}^{x_{n}} \int_{\mathbb{R}^{n-1}} \partial_{x_{i}} \partial_{z_{n}} N(x-z) \\
& \quad \cdot g(z) d z=-\int_{0}^{x_{n}} \int_{\mathbb{R}^{n-1}} \partial_{x_{i}} N(x-z) \partial_{z_{n}} g(z) d z+\frac{\delta_{\text {in }}}{2 n} g(x) \\
& \quad+\int_{\mathbb{R}^{n-1}} \partial_{x_{i}} N(\bar{x}-\bar{z}, 0) g\left(\bar{z}, x_{n}\right) \\
& \cdot d \bar{z}-\int_{\mathbb{R}^{n-1}} \partial_{x_{i}} N\left(\bar{x}-\bar{z}, z_{n}\right) g(\bar{z}, 0) d \bar{z} .
\end{aligned}
$$

Therefore, we arrive at

$$
\begin{aligned}
& \partial_{x_{n}} \int_{0}^{x_{n}} \int_{\mathbb{R}^{n-1}} \partial_{x_{i}} N(x-z) \Gamma\left(z-y^{*}, t\right) \\
& \cdot d z=\int_{0}^{x_{n}} \int_{\mathbb{R}^{n-1}} \partial_{x_{i}} N(x-z) \partial_{z_{n}} \Gamma\left(z-y^{*}, t\right) \\
& \cdot d z+\int_{\mathbb{R}^{n-1}} \partial_{x_{i}} N\left(\bar{x}-\bar{z}, x_{n}\right) \Gamma\left(\bar{z}-\bar{y}, y_{n}, t\right) d \bar{z} .
\end{aligned}
$$


Since

$$
\begin{gathered}
\partial_{y_{n}} \Gamma_{t}(x-y)=-\partial_{x_{n}} \Gamma_{t}(x-y), \\
\partial_{y_{n}} \Gamma_{t}\left(x-y^{*}\right)=\partial_{x_{n}} \Gamma_{t}\left(x-y^{*}\right),
\end{gathered}
$$

therefore, after differentiating $G_{i j}$ with respect to $x_{n}$ variable, we have

$$
\begin{aligned}
\partial_{x_{n}} G_{i j}(x, y, t)= & -\delta_{i j} \partial_{y_{n}}\left[\Gamma(x-y, t)+\Gamma\left(x-y^{*}, t\right)\right] \\
& +4\left(1-\delta_{j n}\right) \partial_{y_{n}} \partial_{x_{j}} \int_{0}^{x_{n}} \int_{\mathbb{R}^{n-1}} \partial_{x_{i}} N(x-z) \\
& \cdot \Gamma\left(z-y^{*}, t\right) d z+4\left(1-\delta_{j n}\right) \\
& \cdot \partial_{x_{j}} \int_{\mathbb{R}^{n-1}} \partial_{x_{i}} N\left(\bar{x}-\bar{z}, x_{n}\right) \Gamma\left(\bar{z}-\bar{y}, y_{n}, t\right) \\
& \cdot d \bar{z}=\partial_{y_{n}} G_{i j}(x, y, t)-2 \delta_{i j} \partial_{y_{n}} \Gamma(x-y, t) \\
& +4\left(1-\delta_{j n}\right) \partial_{x_{j}} \int_{\mathbb{R}^{n-1}} \partial_{x_{i}} N\left(\bar{x}-\bar{z}, x_{n}\right) \\
& \cdot \Gamma\left(\bar{z}-\bar{y}, y_{n}, t\right) d \bar{z} .
\end{aligned}
$$

\section{B. Proof of Proposition 3}

Define a smooth cut-off function $\phi$ such that $\phi(r)=1$ if $0 \leq$ $r \leq 1$ and 0 if $r \geq 2$ with $\int_{0}^{2} \phi(r) d r=1$. For $x \in \mathbb{R}_{+}^{n}$ and $\varepsilon<x_{n} /$ 2 , also define

$$
\phi_{\varepsilon, x}(z)=\phi\left(\frac{|x-z|}{\varepsilon}\right) .
$$

Then, $\phi_{\varepsilon, x}$ is compactly supported in $\mathbb{R}_{+}^{n}$. Let us define

$$
v_{\varepsilon}(x)=\int_{0}^{x_{n}} \int_{\mathbb{R}^{n-1}} \phi_{\varepsilon, x}(z) \partial_{x_{i}} N(x-z) f(z) d z
$$

Differentiating with respect to $x_{j}$ for $j \neq n$ yields

$$
\partial_{x_{j}} v_{\varepsilon}(x)=\int_{0}^{x_{n}} \int_{\mathbb{R}^{n-1}}\left[\partial_{x_{j}} \phi_{\varepsilon, x}(z) \partial_{x_{i}} N(x-z)+\phi_{\varepsilon, x}(z) \partial_{x_{i}} \partial_{x_{j}} N(x-z)\right] f(z) d z .
$$

If $j=n$, we have

$$
\begin{aligned}
& \partial_{x_{n}} v_{\varepsilon}(x)=\int_{0}^{x_{n}} \int_{\mathbb{R}^{n-1}}\left[\partial_{x_{n}} \phi_{\varepsilon, x}(z) \partial_{x_{i}} N(x-z)\right. \\
& \left.\quad+\phi_{\varepsilon, x}(z) \partial_{x_{i}} \partial_{x_{n}} N(x-z)\right] f(z) \\
& \quad \cdot d z+\int_{\mathbb{R}^{n-1}} \phi_{\varepsilon, x}\left(\bar{z}, x_{n}\right) \partial_{x_{i}} N(\bar{x}-\bar{z}, 0) f\left(\bar{z}, x_{n}\right) d \bar{z}
\end{aligned}
$$

Let us set

$$
\begin{aligned}
& I_{1, \varepsilon}(x):= \int_{0}^{x_{n}} \int_{\mathbb{R}^{n-1}} \partial_{x_{j}} \phi_{\varepsilon, x}(z) \partial_{x_{i}} N(x-z) f(z) d z \text { and } I_{2, \varepsilon} \\
&:=\int_{0}^{x_{n}} \int_{\mathbb{R}^{n-1}} \phi_{\varepsilon, x}(z) \partial_{x_{i} x_{j}}^{2} N(x-z) f(z) d z .
\end{aligned}
$$

It is clear that

$$
\lim _{\varepsilon \longrightarrow 0} I_{2, \varepsilon}=\int_{0}^{x_{n}} \int_{\mathbb{R}^{n-1}} \partial_{x_{i} x_{j}}^{2} N(x-z) f(z) d z .
$$

Let us denote $I_{1, \varepsilon}=I_{1, \varepsilon}^{*}+I_{1, \mathcal{\varepsilon}}^{* *}$ where

$$
I_{1, \mathcal{\varepsilon}}^{*}(x):=\int_{0}^{x_{n}} \int_{\mathbb{R}^{n-1}} \partial_{x_{j}} \phi_{\varepsilon, x}(z) \partial_{x_{i}} N(x-z)[f(z)-f(x)] d z,
$$

and

$$
I_{1, \varepsilon}^{* *}:=f(x) \int_{\mathbb{R}^{n-1}} \partial_{x_{j}} \phi_{\varepsilon, x}(z) \partial_{x_{i}} N(x-z) d z .
$$

Observe that $I_{1, \varepsilon}^{*}(x) \longrightarrow 0$ as $\varepsilon \longrightarrow 0$, since

$$
\begin{gathered}
\left|I_{1, \varepsilon}^{*}(x)\right| \leq \int_{|x-z| \leq \varepsilon}\left|\partial_{x_{j}} \phi_{\varepsilon, x}(z)\right|\left|\partial_{x_{i}} N(x-z)\right||f(z)-f(x)| \\
\cdot d z \leq \int_{|x-z| \leq \varepsilon} \frac{1}{\varepsilon} \frac{1}{|x-z|^{n-1}}|x-z|^{\alpha} d z[f]_{\alpha} \leq \varepsilon^{\alpha}[f]_{\alpha}
\end{gathered}
$$

where

$$
[f]_{\alpha}=\sup _{x, z} \frac{|f(x)-f(z)|}{|x-z|^{\alpha}}
$$

Next, we will show that

$$
\begin{aligned}
& \int_{0}^{x_{n}} \int_{\mathbb{R}^{n-1}} \partial_{x_{j}} \phi_{\varepsilon, x}(z) \partial_{x_{i}} N(x-z)
\end{aligned}
$$

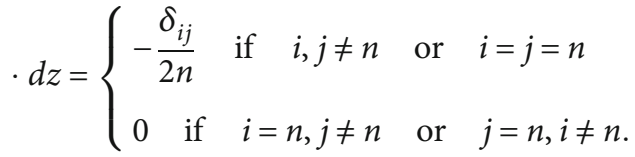

Let us apply a change of variables and let $\varepsilon \longrightarrow 0$, we get

$$
\begin{aligned}
\int_{0}^{x_{n}} \int_{\mathbb{R}^{n-1}} \partial_{x_{j}} \phi_{\varepsilon, x}(z) \partial_{x_{i}} N(x-z) \\
\cdot d z=\frac{1}{\varepsilon} \int_{0}^{x_{n}} \int_{\mathbb{R}^{n-1}} \frac{\left(z_{i}-x_{i}\right)\left(z_{j}-x_{j}\right)}{n \omega_{n}|x-z|^{n+1}} \phi^{\prime}\left(\frac{|x-z|}{\varepsilon}\right) \\
\cdot d z \longrightarrow \int_{0}^{\infty} \int_{\mathbb{R}^{n-1}} \frac{w_{i} w_{j}}{n \omega_{n}|w|^{n+1}} \phi^{\prime}(|w|) d w .
\end{aligned}
$$


If $i, j \neq n$ or $i=j=n$, then by the symmetry of $\left(w_{i} w_{j}\right)$ $\left.|w|^{n+1}\right) \phi^{\prime}(|w|)$ in terms of $w_{n}$ variables, we obtain

$$
\begin{aligned}
& \int_{0}^{\infty} \int_{\mathbb{R}^{n-1}} \frac{w_{i} w_{j}}{n \omega_{n}|w|^{n+1}} \phi^{\prime}(|w|) d w=\frac{1}{2} \int_{\mathbb{R}^{n}} \frac{w_{i} w_{j}}{n \omega_{n}|w|^{n+1}} \phi^{\prime}(|w|) d w \\
& \quad= \frac{1}{2 n \omega_{n}} \int_{0}^{2} \int_{\mathbb{S}^{n-1}} r w_{i} w_{j} \phi^{\prime}(r) d S_{w} d r=-\frac{1}{2 n} \delta_{i j} \int_{0}^{2} \phi^{\prime}(r) d r \\
& \quad=-\frac{1}{2 n} \delta_{i j} .
\end{aligned}
$$

If $i=n, j \neq n$ or $j=n, i \neq n$, then by the antisymmetry of $\left(w_{i} w_{j} /|w|^{n+1}\right) \phi^{\prime}(|w|)$ in terms of $w_{n}$ variables, we obtain

$$
\int_{0}^{\infty} \int_{\mathbb{R}^{n-1}} \frac{w_{i} w_{j}}{|w|^{n+1}} \phi^{\prime}(|w|) d w=0
$$

This completes the proof of Proposition 3.

\section{Proof of Proposition 5}

Denote

$$
\begin{aligned}
I & =\int_{0}^{x_{n} / 2} \int_{\mathbb{R}^{n-1}} \partial_{x_{i} x_{j}}^{2} N(x-z) f(z, y) d z, \\
I I & =\int_{x_{n} / 2}^{x_{n}} \int_{\mathbb{R}^{n-1}} \partial_{x_{i} x_{j}}^{2} N(x-z)\left[f(z, y)-f\left(\bar{x}, z_{n}, y\right)\right] d z, \quad \text { and } \\
I I I & =\int_{x_{n} / 2}^{x_{n}} f\left(\bar{x}, z_{n}, y\right) \int_{\mathbb{R}^{n-1}} \partial_{x_{i} x_{j}}^{2} N(x-z) d \bar{z} d z_{n} .
\end{aligned}
$$

Then

$$
\int_{0}^{x_{n}} \int_{\mathbb{R}^{n-1}} \partial_{x_{i} x_{j}}^{2} N(x-z) f(z, y) d z=I+I I+I I I
$$

Notice that

$\left|\partial_{x_{i} x_{j}}^{2} N(x-z)\right| \leq C|x-z|^{-n}, \quad \int_{\mathbb{R}^{n-1}}|x-z|^{-n} d \bar{z}=C\left|x_{n}-z_{n}\right|^{-1}$.

Then, we have

$$
\begin{aligned}
& \int_{\mathbb{R}_{+}^{n}} I d y \leq C\left(\int_{0}^{x_{n} / 2} \frac{1}{x_{n}-z_{n}} d z_{n}\right)\left(\sup _{z \in \mathbb{R}_{+}^{n}}|f(z, y)| d y\right) \\
& \quad \leq C \sup _{z \in \mathbb{R}_{+}^{n}} \int_{\mathbb{R}_{+}^{n}}|f(z, y)| d y .
\end{aligned}
$$

Since, for $0<\alpha<1$

$$
\begin{aligned}
& \left|\partial_{x_{i} x_{j}}^{2} N(x-z)\left[f(z, y)-f\left(\bar{x}, z_{n}, y\right)\right]\right| \\
& \quad \leq C|x-z|^{\alpha}|x-z|^{-n} \frac{\left|f\left(\bar{z}, z_{n}, y\right)-f\left(\bar{x}, z_{n}, y\right)\right|}{|x-z|^{\alpha}} \\
& \quad \leq C\left|x_{n}-z_{n}\right|^{\alpha}|x-z|^{-n} \frac{\left|f\left(\bar{z}, z_{n}, y\right)-f\left(\bar{x}, z_{n}, y\right)\right|}{|\bar{x}-\bar{z}|^{\alpha}},
\end{aligned}
$$

we have

$$
\begin{aligned}
\int_{\mathbb{R}_{+}^{n}}(I I) d y \leq C \int_{x_{n} / 2}^{x_{n}}\left(x_{n}-z_{n}\right)^{\alpha} \int_{\mathbb{R}^{n-1}}|x-z|^{-n} \\
\times\left[\sup _{\bar{z} \in \mathbb{R}^{n-1}} \int_{\mathbb{R}_{+}^{n}} \frac{\left|f\left(\bar{z}, z_{n}, y\right)-f\left(\bar{x}, z_{n}, y\right)\right|}{|\bar{x}-\bar{z}|^{\alpha}} d y\right] d \bar{z} d z_{n} \leq C x_{n}^{\alpha+1} \\
\quad \times\left(\int_{\mathbb{R}^{n-1}}|x-z|^{-n} d \bar{z}\right)\left(\sup _{x_{n} / 2<z_{n}<x_{n} \bar{z} \in \mathbb{R}^{n-1}} \sup _{\mathbb{R}_{+}^{n}} \frac{\left|f\left(\bar{z}, z_{n}, y\right)-f\left(\bar{x}, z_{n}, y\right)\right|}{|\bar{x}-\bar{z}|^{\alpha}} d y\right) \\
\leq C x_{n}^{\alpha}\left(\sup _{x_{n} / 2<z_{n}<x_{n} \bar{z} \in \mathbb{R}^{n-1}} \sup _{\mathbb{R}_{+}^{n}} \frac{\left|f\left(\bar{z}, z_{n}, y\right)-f\left(\bar{x}, z_{n}, y\right)\right|}{|\bar{x}-\bar{z}|^{\alpha}} d y\right) .
\end{aligned}
$$

Next, we want to show $(I I I)=0$. For that, notice

$$
P V \int_{\mathbb{R}^{n-1}} \partial_{y_{i} y_{j}}^{2} N(x-y) d \bar{y}=\lim _{\varepsilon \longrightarrow 0, R \longrightarrow \infty} \int_{\varepsilon \leq|\bar{x}-\bar{y}| \leq R} \partial_{y_{i} y_{j}}^{2} N(x-y) d S_{\bar{y}},
$$

and

$$
\begin{aligned}
& \int_{\varepsilon \leq|\bar{x}-\bar{y}| \leq R} \partial_{y_{i} y_{j}}^{2} N(x-y) d \bar{y}=\int_{S_{R}(\bar{x})} \partial_{y_{i}} N(x-y) n_{j} d S_{\bar{y}} \\
& -\int_{S_{\varepsilon}(\bar{x})} \partial_{y_{i}} N(x-y) n_{j} d S_{\bar{y}} .
\end{aligned}
$$

Here, $S_{R}(\bar{x})=\left\{\bar{y} \in \mathbb{R}^{n-1}:|\bar{x}-\bar{y}|=R\right\}$, and $n_{j}=y_{j}-x_{j} / \mid \bar{y}$ $-\bar{x} \mid$ is the $j$ th component of the unit outer normal vector. If $i=1, \cdots, n-1$, then

$$
\begin{aligned}
& \left|\int_{S_{R}(\bar{x})} \partial_{y_{i}} N(x-y) n_{j} d S_{\bar{y}}\right|=\left|\int_{S_{R}(\bar{x})} \frac{y_{i}-x_{i}}{|x-y|^{n}} \frac{y_{j}-x_{j}}{|\bar{y}-\bar{x}|} d S_{\bar{y}}\right| \\
& \quad=\left|\int_{S_{n-2}} \frac{w_{i} w_{j}}{n \omega_{n}\left(R^{2}+\left(x_{n}-y_{n}\right)^{2}\right)^{n / 2}} R^{n-1} d S_{w}\right| \\
& \quad \leq \frac{C}{R} \longrightarrow 0 \text { as } R \longrightarrow \infty,
\end{aligned}
$$


where $S_{n-2}$ is the unit sphere in $\mathbb{R}^{n-1}, w_{i}=y_{i} /|y|$ is the outward unit normal vector to $S_{n-2}$, and

$$
\begin{aligned}
& \left|\int_{S_{\varepsilon}(\bar{x})} \partial_{y_{i}} N(x-y) n_{j} d S_{\bar{y}}\right|=\left|\int_{S_{n-2}} \frac{w_{i} w_{j}}{n \omega_{n}\left(\varepsilon^{2}+\left(x_{n}-y_{n}\right)^{2}\right)^{n / 2}} \varepsilon^{n-1} d S_{w}\right| \\
& \quad \leq C \frac{\varepsilon^{n-1}}{\left|x_{n}-y_{n}\right|^{n}} \longrightarrow 0 \text { as } \varepsilon \longrightarrow 0 .
\end{aligned}
$$

If $i=n$, then

$$
\int_{S_{R}(\bar{x})} \partial_{y_{i}} N(x-y) n_{j} d S_{\bar{y}}=0
$$

since

$$
\begin{gathered}
\int_{S_{R}(\bar{x})} \partial_{y_{i}} N(x-y) n_{j} d S_{\bar{y}}=\int_{S_{n-2}} \frac{\left(x_{n}-y_{n}\right) w_{j}}{n \omega_{n}\left(R^{2}+\left(x_{n}-y_{n}\right)^{2}\right)^{n / 2}} R^{n-2} d S_{w} \\
=\frac{\left(x_{n}-y_{n}\right) R^{n-2}}{n \omega_{n}\left(R^{2}+\left(x_{n}-y_{n}\right)^{2}\right)^{n / 2}} \int_{S_{n-2}} w_{j} d S_{w}=0 .
\end{gathered}
$$

Similarly

$$
\int_{S_{\varepsilon}(\bar{x})} \partial_{y_{i}} N(x-y) d \bar{y}=0
$$

This implies that

$$
\int_{\mathbb{R}^{n-1}} \partial_{y_{i} y_{j}}^{2} N(x-y) d \bar{y}=0,
$$

Hence, we finally show that $(I I I)=0$.

\section{Data Availability}

I have provided all the essential references that I have used in this research article in the reference section.

\section{Conflicts of Interest}

The authors declare that they have no conflicts of interest.

\section{References}

[1] T. Kato, "Strong $L^{p}$-solutions of the Navier-Stokes equation in $R^{m}$, with applications to weak solutions," Mathematische Zeitschrift, vol. 187, no. 4, pp. 471-480, 1984.

[2] M. Wiegner, "The Navier-Stokes equations-a neverending challenge?," DMV Jahresbericht, vol. 101, Band Heft 1, pp. 1-25, 1999.

[3] H. Amann, "On the Strong Solvability of the Navier-Stokes Equations," Journal of Mathematical Fluid Mechanics, vol. 2, no. 1, pp. 16-98, 2000.

[4] J. R. Cannon and G. H. Knightly, "A Note on the Cauchy Problem for the Navier-Stokes Equations," SIAM Journal on Applied Mathematics, vol. 18, no. 3, pp. 641-644, 1970.
[5] N. W. Kim and D. H. Chae, "On the uniqueness of the unbounded classical solutions of the Navier-Stokes and associated equations," Journal of Mathematical Analysis and Applications, vol. 186, no. 1, pp. 91-96, 1994.

[6] G. P. Galdi and P. Maremonti, "A uniqueness theorem for viscous fluid motions in exterior domains," Archive for Rational Mechanics and Analysis, vol. 91, no. 4, pp. 375-384, 1986.

[7] Y. Giga, K. Inui, J. Kato, and S.'y. Matsui, "Remarks on the uniqueness of bounded solutions of the Navier-Stokes equations," Nonlinear Analysis, vol. 47, no. 6, pp. 4151-4156, 2001.

[8] H.-O. Bae and B.-J. Jin, "Existence of strong mild solution of the navier-stokes equations in the half space with nondecaying initial data," Journal of the Korean Mathematical Society, vol. 49, no. 1, pp. 113-138, 2012.

[9] V. A. Solonnikov, "On nonstationary Stokes problem and Navier-Stokes problem in a half- space with initial data nondecreasing at infinity," Problemy Matematisheskogo Analiza, vol. 25, pp. 189-210, 2003.

[10] S. Pathak, "A priori estimates in terms of the maximum norm for the solution of the Navier-Stokes equations with periodic initial data," The Nepali Mathematical Sciences Report, vol. 36, no. 1-2, pp. 39-50, 2020.

[11] S. Pathak, " $L^{\infty}$-norm estimates of the solution for the incompressible Navier-Stokes equations for non-decaying initial data," International Journal of Mathematical Analysis, vol. 14, no. 2, pp. 91-107, 2020.

[12] S. Ukai, "A solution formula for the Stokes equation in $\mathbb{R}_{+}^{n}$," Communications on Pure and Applied Mathematics, vol. 40, no. 5, pp. 611-621, 1987.

[13] W. Borchers and T. Miyakawa, " $L^{2}$ decay for the Navier-Stokes flow in halfspaces," Mathematische Annalen, vol. 282, no. 1, pp. 139-155, 1988.

[14] Y. Fujigaki and T. Miyakawa, "Asymptotic profiles of nonstationary incompressible Navier-Stokes flows in the half-space," Methods and Applications of Analysis, vol. 8, no. 1, pp. 121158, 2001.

[15] Y. Giga, S.'y. Matsui, and Y. Shimizu, "On estimates in Hardy spaces for the Stokes flow in a half space," Mathematische Zeitschrift, vol. 231, no. 2, pp. 383-396, 1999.

[16] Y. Shimizu, " $L$ estimate of the first-order space derivatives of Stokes flow in a half space," Funkcial Ekvac, vol. 42, no. 2, pp. 291-309, 1999.

[17] P. Maremonti and G. Starita, "Nonstationary Stokes equations in a half-space with continuous initial data," Journal of Mathematical Sciences, vol. 127, no. 2, pp. 1886-1914, 2005.

[18] V. A. Solonnikov, "On estimates of solutions of the nonstationary Stokes problem in anisotropic Sobolev spaces and on estimates for the resolvent of the Stokes operator," Uspekhi Mat. Nauk, vol. 58, no. 2(350), pp. 123-156, 2003. 